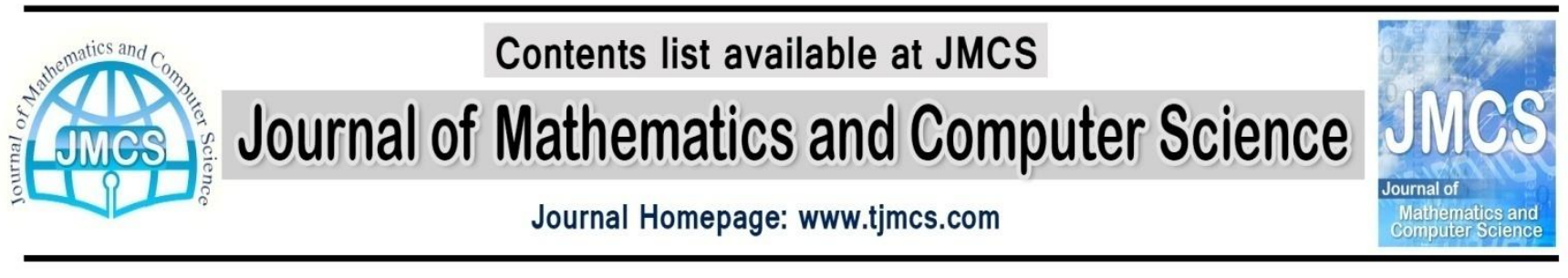

\title{
Fairness Aware Downlink Scheduling Algorithm for LTE Networks
}

\author{
Mahnaz Sotoudeh Bahreyni, Vahid Sattari-Naeini \\ Department of Information Technology Engineering, Kerman Science and Research \\ Branch, IslamicAzad University, Kerman, Iran \\ mahnaz.sotoodeh@gmail.com \\ Department of Computer Engineering, ShahidBahonar University of Kerman, Kerman, \\ Iran \\ vsnaeini@uk.ac.ir
}

\author{
Article history: \\ Received: April 2014 \\ Accepted: May 2014 \\ Available online : June 2014
}

\begin{abstract}
This paper proposes a new scheduling algorithm in downlink Long Term Evolution networks whichis adoptable with fast variations in channel conditions.It allocates resources in a fair manner among users so that it increases cell edge users' performance. Besides, it makes a good trade-off between throughput and fairness. The proposed method is simulated and compared with three scheduling algorithms available for Long Term Evolution. The results showgood degree of fairness at the cost of small decrease in amount of system throughput.
\end{abstract}

Keywords: Downlink,Radio Resource Allocation, Fairness, LTE.

\section{Introduction}

LTE(Long-Term Evolution) is one of the most promising standards for the fourth generation (4G) wireless networks [1]. It is an evolution of the Universal Mobile Telecommunications System (UMTS) standardized by the 3rd Generation Partnership Project (3GPP) in its $8^{\text {th }}$ release for the development of wireless broadband networks with very high data rates[2]. LTE provides better services to mobile users who need a lot of bandwidth for multimedia applications such as live streaming, online gaming, and mobile TV. Technically, LTE provides a high data rate and can operate in different bandwidths ranging from $1.4 \mathrm{MHz}$ to $20 \mathrm{MHz}$. Indeed, advantages of LTE 
design arehigher user bit rates, lower delays, increased spectrum efficiency, reduced cost, and operational simplicity. To gain these goals LTE uses several technologies, which include Orthogonal Frequency Division Multiple Access (OFDMA) [3], Single Carrier Frequency Division Multiple Access (SC-FDMA) [4] and Multiple Input Multiple Output (MIMO)[5]. LTE uses OFDMA for downlink and SC-FDMA for uplink transmission [6]. To optimize system performance, scheduling divides and allocates radio resources among different users simultaneously, keeping quality of service (QoS).

In LTE networks, the role of resource scheduling is very important.Because, agood performance can be achievedby a suitable assignment of radio resources to each user. Thereforescheduling is a very important Radio Resource Management (RRM) mechanism. Designing a downlink scheduling algorithm provides challenges such as maximization of the system capacity, spectral efficiency, fairness provisioning, and error rate consideration. In [7], the spectral efficiencies and cell-edge user throughputs for three combinations of different packet scheduler, namely TDBET/FD-TTA, TD-PF/FD-PF, and TDMT/FD-MT, are compared with those for a reference Round Robin scheduler in which one user scheduled per Packet scheduler (PS). As a result, the benefits of multiuser diversity and channel dependent scheduling have been investigated. In [6],a Fair Downlink Scheduling algorithm is proposed. The algorithm makes use of Assignment Model for resource allocation to all the selected users during each Transmission Time Interval (TTI). The proposed algorithm is compared with three scheduling algorithms Round Robin, Best CQI, and MS Algo. MS Algo is a proposed algorithm investigated in [8]. In [9], a new scheduling algorithm was proposed that resolves trade-off between throughput and fairness. The proposed algorithm has been tested in different scenarios and compared with Round Robin and Best CQI scheduling algorithms. In $[10,11]$, scheduling strategies based on Proportional Fair algorithm have been proposed, which aim to increase users' throughput and maintain fairness among them. In [12], a scheduler which takes into account the status of User Equipment (UE) buffers, in addition to channel conditions, is proposed to reduce packet loss rate due to buffer overflow while maintaining a high system throughput and good fairness among users.

In this paper a new scheduling algorithm is introduced. This algorithm provides fairness in resource allocation and at the same time increases the system capacity within the cell, and makes a good trade-off between throughput and fairness. Fairness means farthest users can gain the resources in a fairly manner to achieve better performance.

In section 2 the LTE downlink framework is described. After that theproposed scheduling algorithm along with some other the LTE scheduling strategies are presented. Later on, Section 4 explains the simulation parameters. In section 5 simulationis performed. The performance of our algorithm is evaluated and compared with three well-known algorithms: Round Robin, Best CQI, and Proportional Fair. Finally, Section 6 concludes the paper.

\section{LTE frame structure}

In LTE, downlink and uplink transmissions are grouped into radio frames of length 10 milliseconds (ms). Each radio frame is divided into 10 subframes of $1 \mathrm{~ms}$ duration, which each subframe is further divided into 2 slots. Each slot consists of 7 or 6 OFDM symbols for normal or extended cyclic prefix, respectively. The LTE frame structure is illustrated in Figure 1. The smallest modulation structure in LTE is one symbol in time vs. one subcarrier in frequency and is called a Resource Element (RE). REs are further aggregated into Resource Blocks (RB), with the typical RB having dimensions of 7 symbols by 12 subcarriers. The RE and RB structuresare also shown in Figure 1The number of symbols in a RB depends on the Cyclic Prefix (CP) in use. During the use of normal CP, the RB contains seven symbols, whereas in the case of extended CP, which is used due to extreme delay spread or multimedia broadcast modes, the RB contains six symbols [13]. 


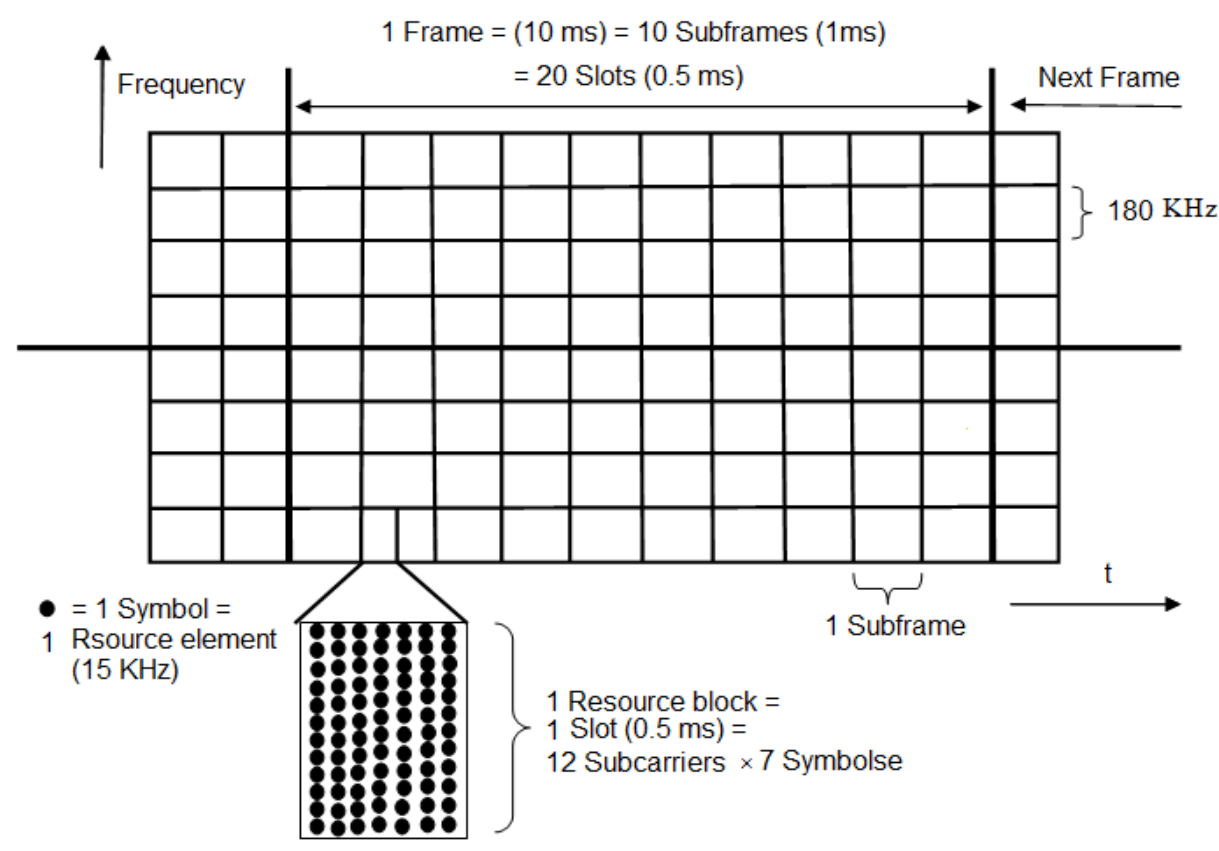

Figure 1.LTE frame structure [2]

\section{LTE downlink scheduling}

\subsection{Round Robin scheduling (RR)}

Round Robin scheduling is a non-aware scheduling scheme that lets users take turns in using the shared resources (time/RBs), without taking the instantaneous channel conditions into account. Therefore, it offers great fairness among the users in radio resource assignment, but degrades the system throughput [14].Figure 2 shows an example of RR algorithm, which means that each user will be placed in a queue. The algorithm selects the users without considering channel condition. If all the users have been served, the scheduler will start again with the same queue.

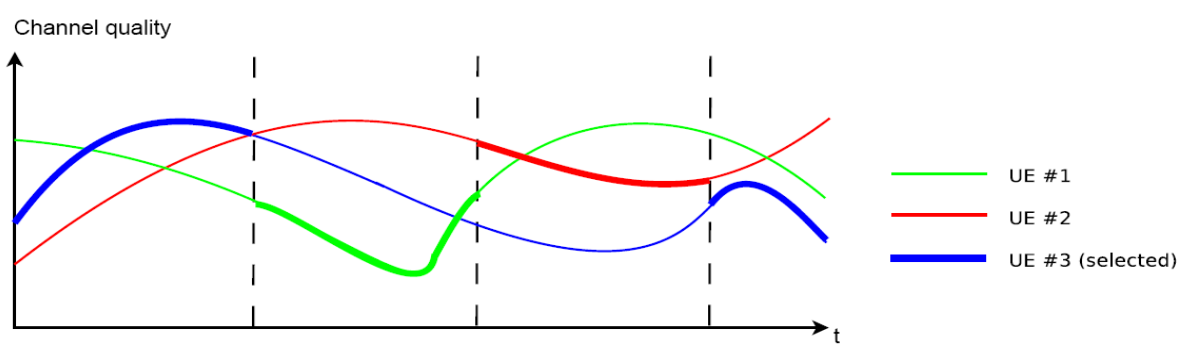

Figure 2.Example of a Round Robin scheduler [15]

The major advantage of this kind of algorithm is its simplicity. Major disadvantage of RR algorithm is that, this algorithm doesn't consider users' CQI feedback, in which it leads to low and unequal throughput.

\subsection{Best CQI scheduling}

This scheduling algorithm allocates resources to those users with best channel condition.For this, users should send CQI feedback to eNodeB. The highest CQI value means best channel 
conditions and the recourses will allocate to this users. In this strategy the users who are far away from eNodeB (i.e. Cell edge users) don't scheduleas well. Figure 3 shows an example of BEST CQI algorithm. This figure shows what happens if a user is near to the base station; therefore, this user has better channel condition rather than others. So scheduling algorithm always schedule this user, it cause other users' starvation.

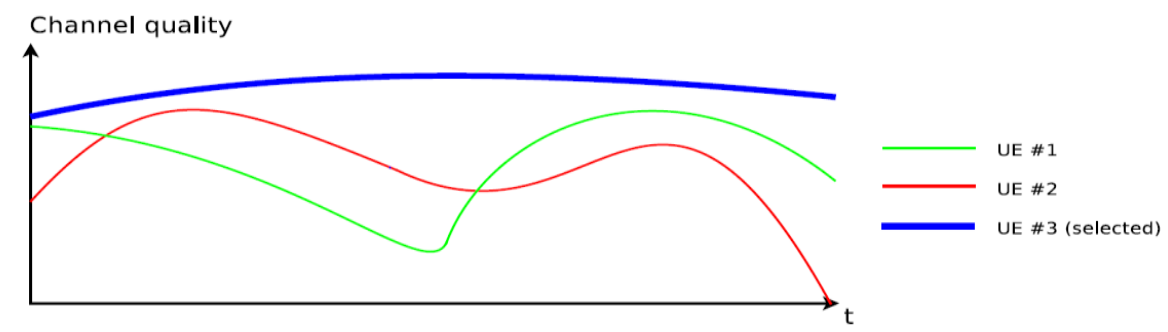

Figure 3. Example of a best CQI scheduler [15]

\subsection{Proportional Fair scheduling (PF)}

This algorithm allocates more resourcesto a user with relatively better channel condition. For scheduling users, this algorithm not only considers channel condition but also tries to maintain fairness among the users. Therefore, the highest throughput of cell together with degree of fairness is provided. The main goal of this algorithm is achieving a balance between highest cell throughput and fairness.

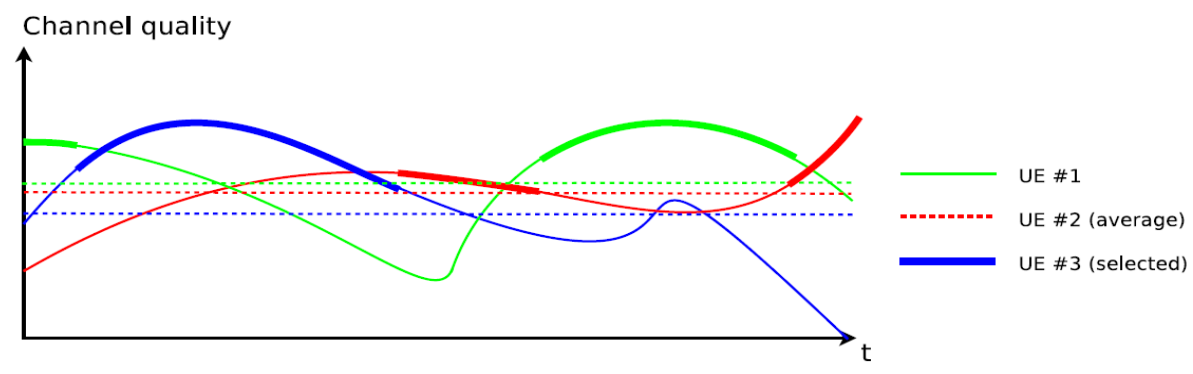

Figure4.Example of a Proportional Fair scheduler [15]

Figure 4 shows an example of a Proportional Fair Scheduler.In this algorithm the user that its data rate is farthest above its average rate will bechosen. Respect to freeness, $\mathrm{PF}$ algorithm is better among two presented algorithms (RR,Best CQI), but it is not enough complete because of some exceptions. For instance, when a user moves closer to eNodeB from a far distance, its SNR will increase constantly (in a scenario without signal shadowing). Therefore this user's actual SNR will be always above its average;accordingly, it is very possible that this user will be often scheduled. On the other hand, if a user goes farther than eNodeB, its actual SNR will always be below its average. Therefore the probability of this user for scheduling is very low and it may lead to starving. PF algorithm provides higher throughput for users near to eNodeB.

\subsection{The proposed downlink scheduling method}

In the new algorithm it is assumed that each eNodeB receives channel feedback information in the form of CQI-feedback matrix. The matrix size equals to (No. of UEs) $\times$ (No. of RBs) in each TTI. The proposed method gives preferences to those users which use less bandwidth than others. Also it evenly distributes the resources among the users during each TTI; therefore, the fairness is 
taken into considerationfor users by the proposed algorithm, and at the same time users' system capacity is increased. The new algorithm operates as follows:

1. For the current TTI, obtain the channel information for different UEs in the form of CQI-Feedback matrix.

2. Calculate the value of parameter $P$ for each user:

$\mathrm{a}=$ expected data rate for the next time interval

$\mathrm{b}=$ Average Throughput

$\mathrm{P}=a / b$

3. Select the users according to parameter $\mathrm{P}$; give preference to those users which have maximum $P$ value; then put them in a FIFO queue.

4. Schedule the user at the front of the queue; move it to the end of queue for scheduling in the next round, during the current TTI.

5. Repeat steps 1 through 4 for each TTI.

The estimated data rate is derived from the actual SNR.In steps 1 to 3 , the algorithm increase system capacity performance within the cellby considering channelconditions and users' requirements. Besides, it maintains fairnessin step 4.The focus of the algorithm is to optimize the resource assignment in scenarios where the number of users is bigger than the number of available RBs. It is considered that these scenarios represent a bigger challenge for the scheduling algorithm.

Throughput Fairness Index is obtained by Jain's equation to calculate fairness among the users, by Equation (1):

$$
\left(x_{1,2}, \ldots, x_{n}\right)=\frac{\left(\sum_{i=1}^{n} x i\right)^{2}}{n \sum_{i=1}^{n} x i^{2}}
$$

In this equation, $\mathrm{n}$ is the number of users and $\mathrm{xi}$ is the throughput for jth user. To reach the highest fairness, when all the users have the same throughput, fairness index is equal to 1 [16].

\section{Simulation parameters}

LTE System Level Simulator [17] is used to evaluate the performance of the four scheduling algorithms, the proposed method, Round Robin, Best CQI, and Proportional Fair. The simulator is MATLAB-based and implements a standard compliant LTE downlink. Simulations are performed for 8, 18, and 27 users which are more than the available RBs. Bandwidths are chosen as 1.4, 3, and $5 \mathrm{MHz}$ respectively. The duration of simulation is equal to 500 TTI. Table 1 summarizes the simulation parameters.

Table 1.Simulation parameters

\begin{tabular}{|c|c|}
\hline Parameter & Value \\
\hline Number of Basestations & 1 \\
\hline Bandwidth (in MHz) & $1.4,3,5 \mathrm{MHz}$ \\
\hline Frequency & $2 \mathrm{GHz}$ \\
\hline Channel type & Pedestrian-B \\
\hline Number of UEs & $8,18,27$ \\
\hline Number of RBs & $6,15,25$ \\
\hline UE speeds of interest & $5 \mathrm{~km} / \mathrm{hr}$ \\
\hline Simulation length & $500 \mathrm{TTI}$ \\
\hline
\end{tabular}




\begin{tabular}{|c|c|}
\hline Scheduling Algorithms & $\begin{array}{c}\text { Round-Robin,Best CQI, } \\
\text { Proportional Fair,Proposed Method }\end{array}$ \\
\hline Transmission Mode & SISO \\
\hline
\end{tabular}

Three multi-user scenarios are chosen for simulation. Distribution of users in each scenario is uniform for all algorithms. Figure 5 shows the distribution of the users in scenario 2. It is notable that in all scenarios users from 1 to the last one have a farther distance from eNodeB respectively.

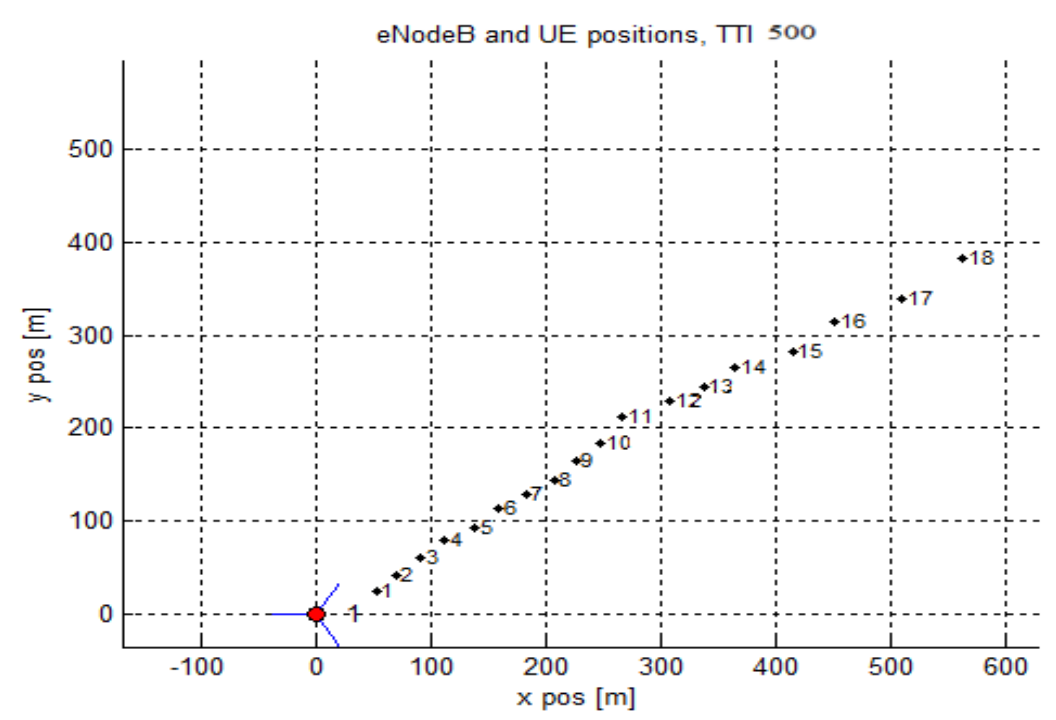

Figure 5.The comparative distance between eNodeB and UE. (reddot represents eNodeB and

\section{Simulation results and discussions} black dots represent UEs)

In this section the performance of the proposed algorithm is evaluated and compared with traditional scheduling algorithms, Proportional Fair (PF), Round Robin (RR), and Best CQI. The considered parameters are:The overall cell throughput for each multiuser scenario,the individual user's throughput for multiuser scenario 2and Fairness. The overall cell throughputs for different scenarios, 8 users at $1.4 \mathrm{MHz}, 18$ users at $3 \mathrm{MHz}$, and 27 users at $5 \mathrm{MHz}$ bandwidths, are shown in Figures 6, 7 and 8respectively. 


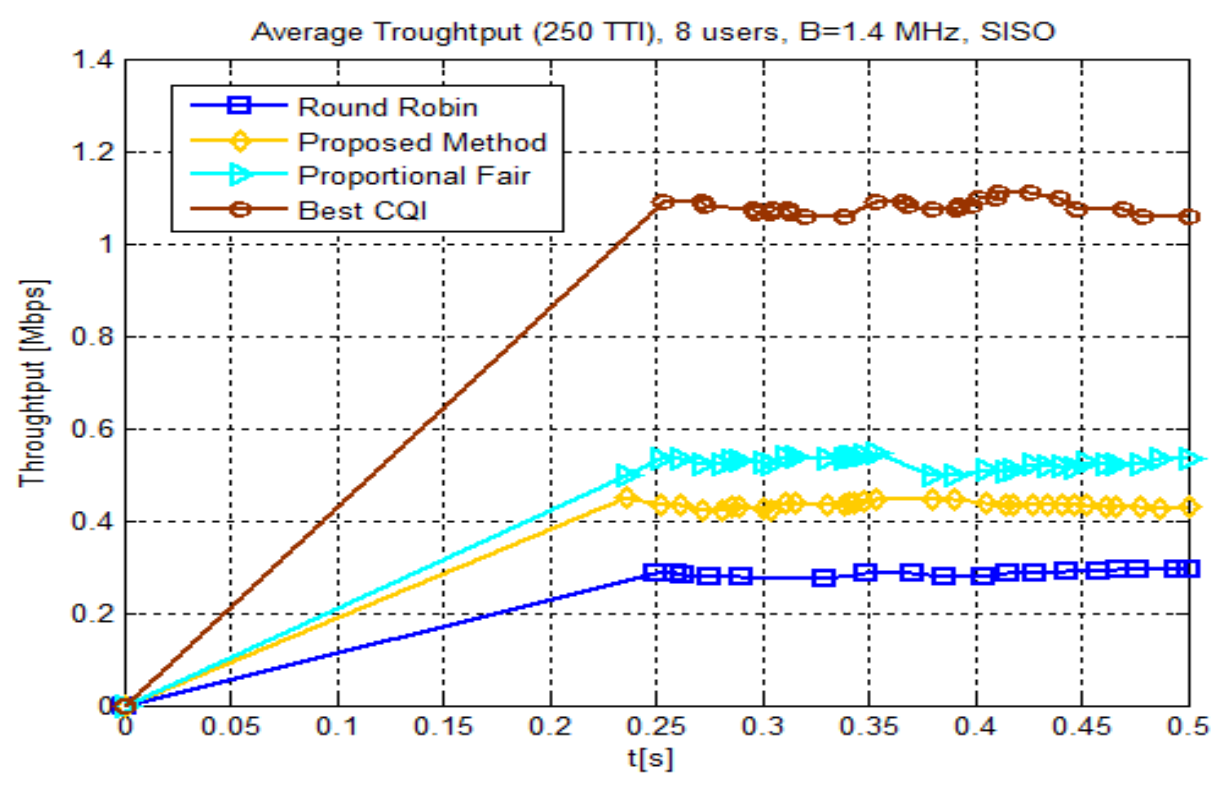

Figure 6.Cell throughput for 8 users, $B W=1.4 \mathrm{MHz}$

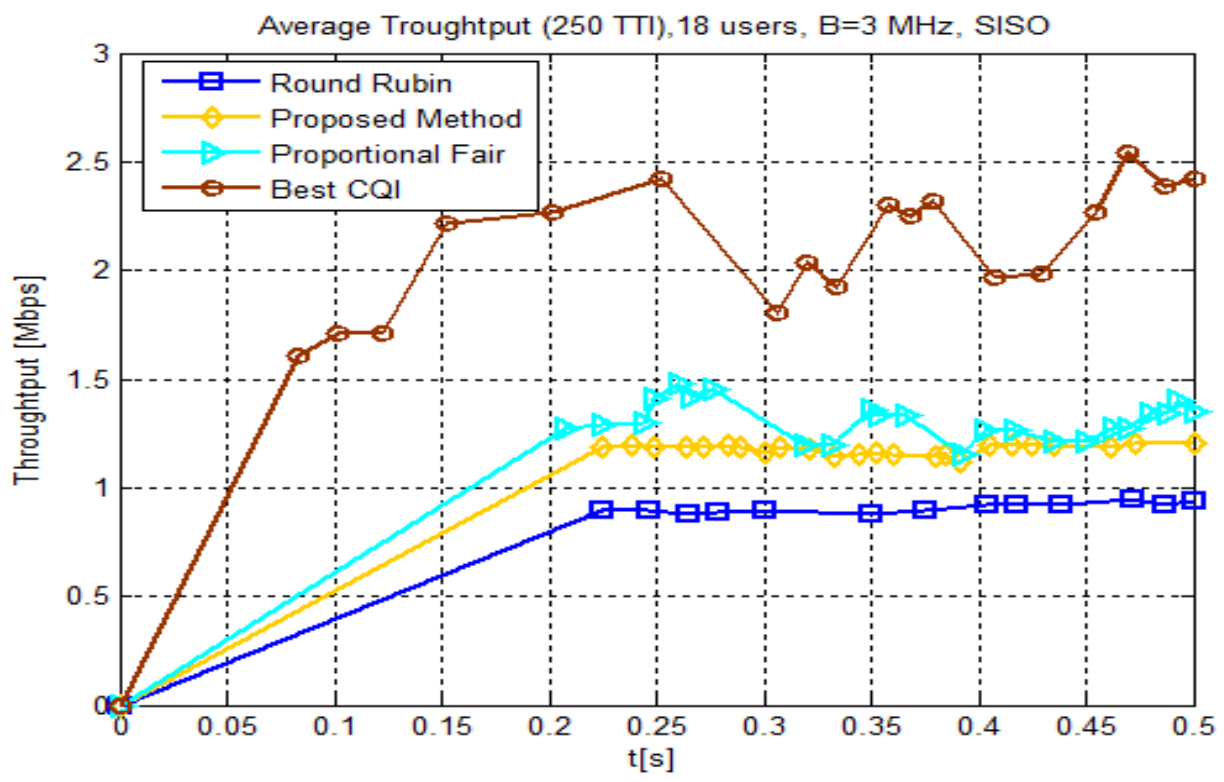

Figure 7.Cell throughput for 18 users, $B W=3 \mathrm{MHz}$ 


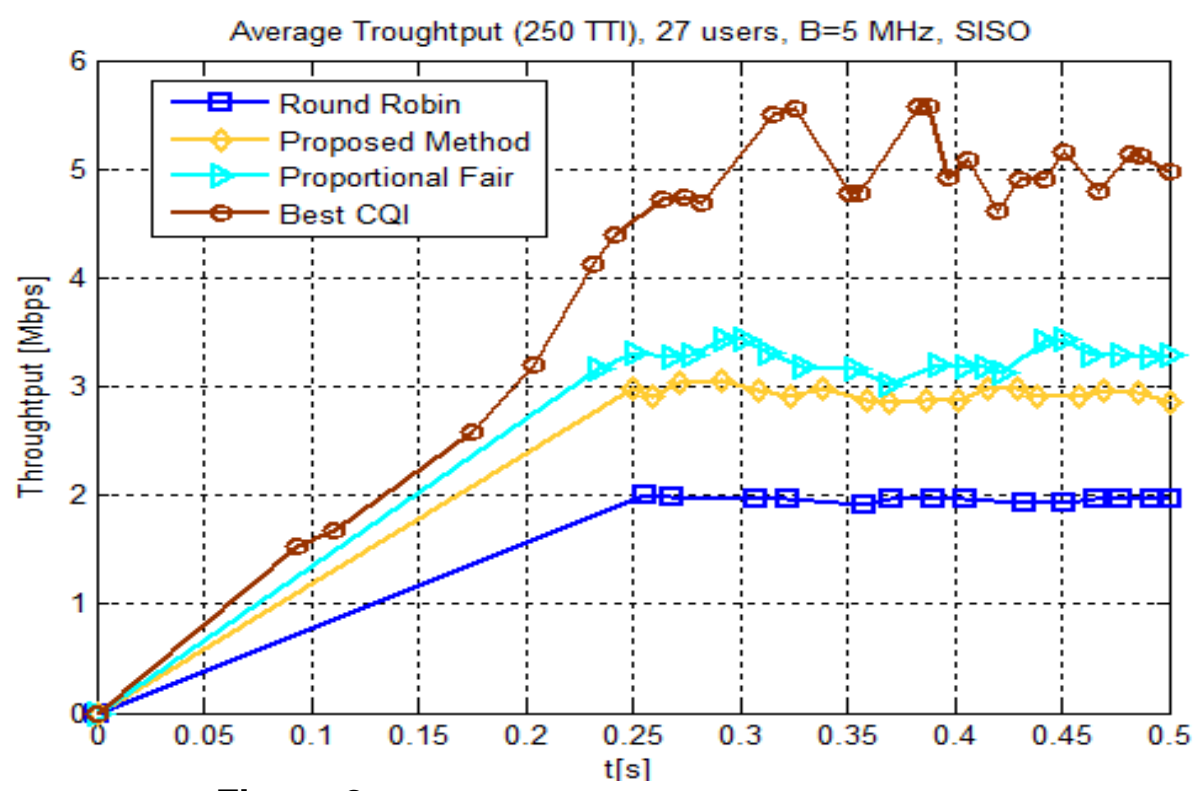

Figure 8.Cell throughput for 27 users, $B W=5 \mathrm{MHz}$

The analysis of curves in Figures 6, 7 and 8 is as follows: In each scheduling algorithm, the amount of variations in system throughput at different timesreveals fairness in resource allocation for all UEs. In all the three scenarios, since the Best CQI scheduler just schedules the users with best channel conditions, it has the high throughput. High variation of its curves demonstrates that distribution of resources among the users is very unfairly. As a consequence, maximizing the throughput results in the degradation of fairness among users.PF algorithm tries to make a balance between fairness and throughput. In orderto increase some user's throughput, it assigns more resources to them. The variation in the curves of this algorithm demonstrates this situation.Simulation results in three scenarios show throughput of the proposed algorithm is about 0.11, less than that of PF one. Because, this algorithm is designed to provide good fairness among the users and achieve the minimum level of QoSfor all users. Therefore, system throughput is decreased. Since the variation in the curve of this algorithm is less than that of PF one,it is expected that the proposed algorithm provides a better fairness. The RR algorithm provides lower throughput for system, but its throughput is more stable than other schedulers. Since this algorithm behaves all users equally without considering channel conditions and their requirements, therefore its system throughput is constant but low. 


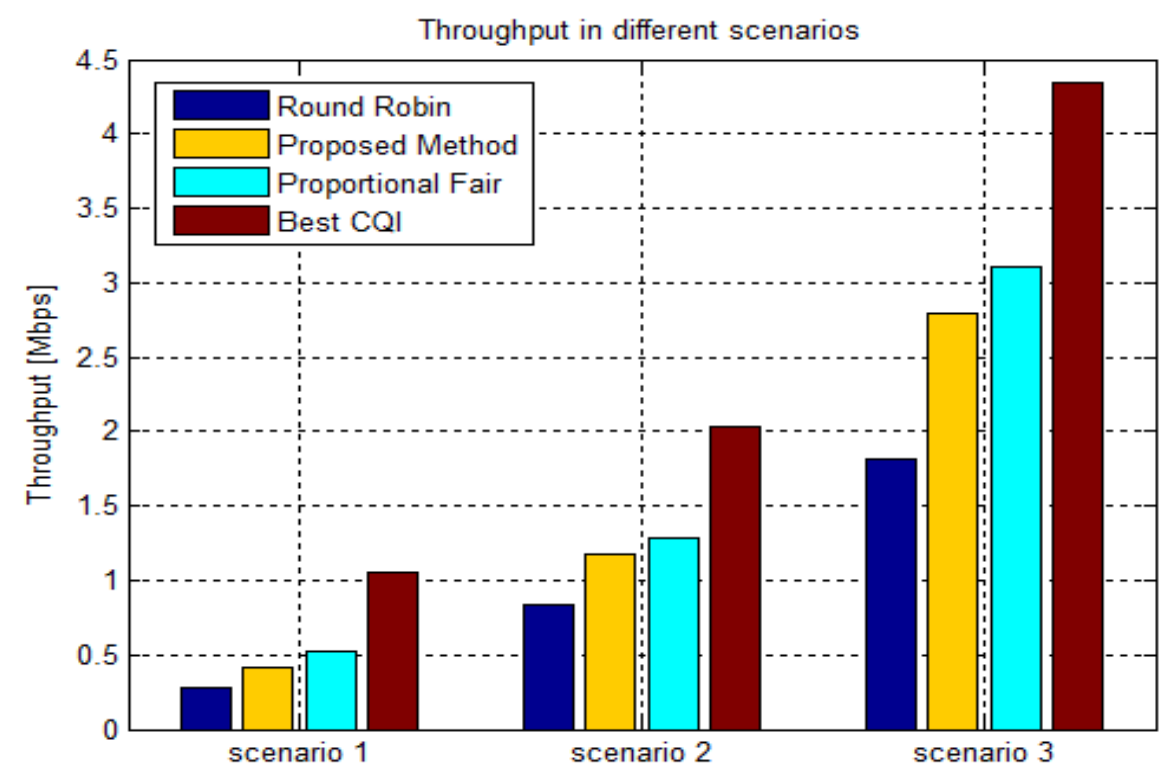

Figure 9.Average throughput in different scenarios

Figure 9 shows average system throughput in different scenarios. In the first scenario, the average system throughput by all algorithms is rather low. It is because of narrower bandwidth. Same kind of characteristics is seen in all the scenarios.

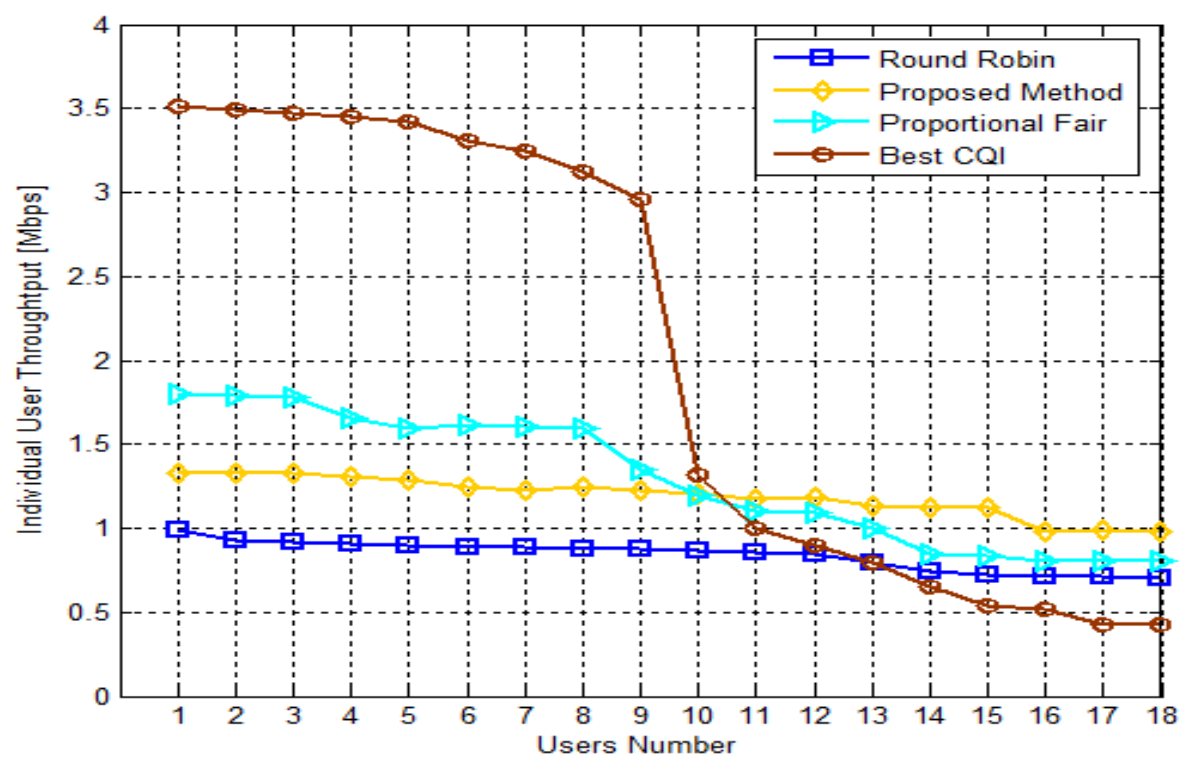

Figure 10. Achievable throughput for each user at different distances from eNodeB

Users' throughput, as a function of distance from eNodeB, is showninFigure10. The results showthat the fairness of the proposed algorithm is equalto 0.8971 , which is higher than 0.7812 values for PF. PF algorithm provides higher throughput for users near to eNodeB. But whatever users' distance from eNodeBis increased, the proposed algorithm performance outperformsthat of PF algorithm, and provides higher throughput for distance users even in poor channel 
conditions;since in the proposed algorithm users with poorest channel conditions are scheduled more rather than PF algorithm by increasing diversities of users in a TTI.

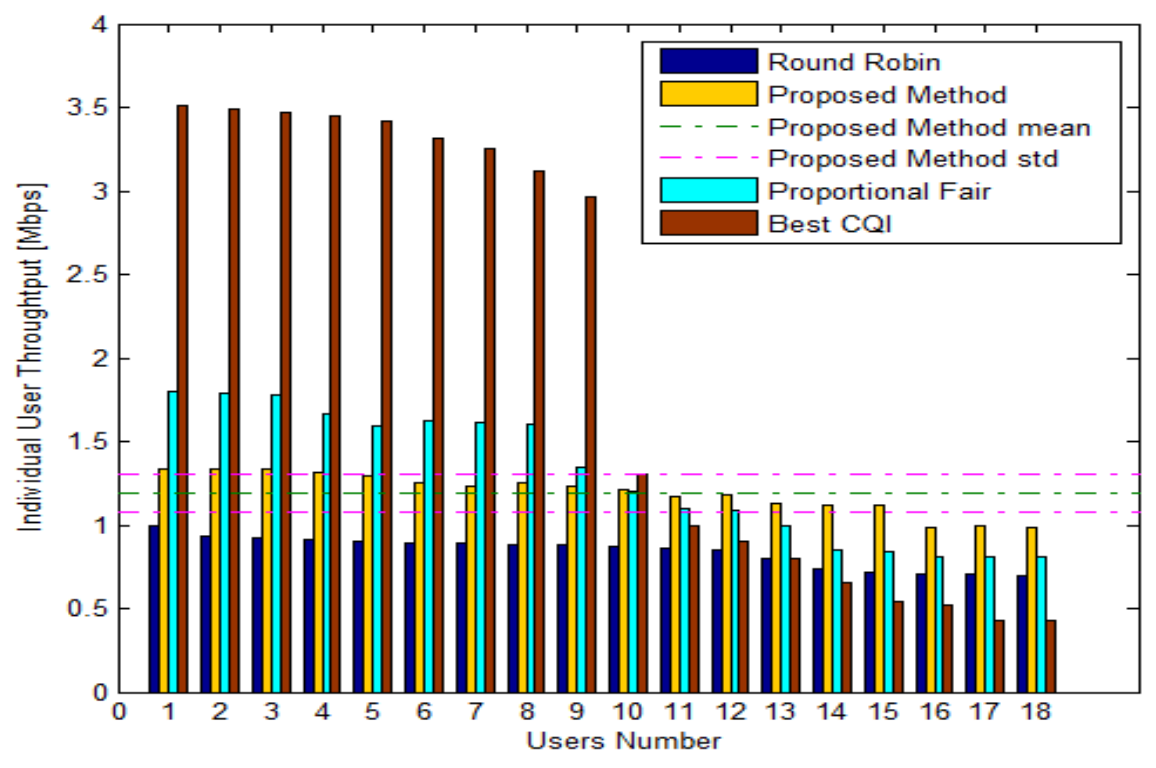

Figure 11.Comparing fairness-throughput for each user at different distance from eNodeB

It is shown in Figure 11 that the average throughput of the proposed algorithm and the standard deviation (STD) are 1.19 and 0.11 , respectively; while these metrics are 2.03 and 1.36 for best CQI algorithm, respectively. PF algorithm provides anaverage throughput of 1.29 and STD of 0.38 . In RR algorithm, average throughput and STD are equal to 0.84 and 0.08 , respectively. So the proposed algorithm, in comparison toother schedulers, provides a balance between throughput and fairness. It is notable that the proposed algorithm is more efficient for cells that their QoS guarantee is more important than increase in capacity of cell.

\section{Conclusion}

This paper proposed a new Downlink scheduling algorithm. Performance of the new algorithm was compared with three well-known scheduling algorithms available for LTE networks.Simulation results showed that the proposed method could provide fairness better than the three mentionedalgorithms; also a better trade-off between fairness and throughput has been obtained. With respect to fairness, best CQI was not a good approach; because in this algorithm, users with bad channel condition were neglected. RR algorithm had the highest factor of fairness, but it neglected users' CQI feedback which led to degradation in system performance. PF algorithm was a good approach; because it considered both channel condition and fairness, but it was not yet perfect because of some exceptions, for example the users far from eNodeB. The proposed algorithm not only peaked highest fairness factor of RR algorithm, but it also considered channel conditions results fair distribution of resources among the users.This causes absolutely poorest channel conditions are less avoided in scheduling such that it could improve cell-edge users' performance.

\section{References}


[1] A. Ghosh, R. Ratasuk, B. Mondal, N. Mangalvedheand, T. Thomas, LTE-Advanced: next-generation wireless broadband technology,ACM Transactions on Multimedia Computing, Communications And ApplicationsVol. 17 No. 3 (2010) 10-22.

[2] M. Sauter, From GSM To LTE: An introduction to mobile networks and mobile broadband, John Wiley \&Sons Ltd., 2011.

[3]. R. Van Nee, R. Prasad, OFDM for wireless multimedia communications, Artech House Publications, 2000.

[4] H. Holma, A. Toskala, LTE for UMTS-OFDMA and SC-FDMA based radio access, John Wiley \&Sons Ltd., 2009.

[5] G. L. Stuber, J. R. Barry, S.W. Mclaughlin, Y. Li, M.A. Ingram, T.G. Pratt, Broadband MIMO-OFDM wireless Communications, Proceedings of IEEEVol. 92 No. 2(2004) 271-294.

[6] A.C.B. Akki, S.M. Chadcha, Fair downlink scheduling algorithm for 3gpp LTE networks, I. J. Computer Network and Information Security vol. 6 (2013) 34-41.

[7] P. Kela, J. Puttonen, N. Kolehmainen, T. Ristaniemi, T. Henttonen, M. Moisio, Dynamic packet scheduling performance in utra long term evolution downlink, In Proceedings of the $3^{\text {rd }}$ International Symposium on Wireless Pervasive Computing (ISWPC 2008) (2008) 308-313.

[8] D. Talevski, L. Gavrilovska,Novel scheduling algorithms for LTE downlink transmission, Telfor Journal 4No.2 (2012) 24-25.

[9] T. Dikamba, Downlink Scheduling In 3GPP Long Term Evolution (LTE), M.Sc. Thesis, Delft University of Technology, Netherlands, 2011.

[10] R. Kwan, C. Leung, J. Zhang, Proportional fair multiuser scheduling in LTE,Proceedings of IEEE Signal Processing LettersVol. 16No.6 (2009) 461-464.

[11] G. Monghal, K.I. Pedersen, I.Z. Kovacs, P. E. Mogensen, Qos oriented time and frequency domain packet schedulers for the UTRAN long term evolution, In Proceedings of IEEE Veh. Tech. Conf., VTC-Spring, Marina Bay, Singapore, (2008).

[12] Y. Lin, G. Yue, Channel-Adapted and buffer-aware packet scheduling in LTE wireless communication system, In Proceedings of the IEEE International Conference on Wireless Communications, Networking and Mobile Computing (Wicom'08), (2008).

[13] D. Mannani, Modeling and simulation of scheduling algorithms in LTE networks, B.sc Thesis,Electrical and Computer Engineering, The Institute Of Telecommunications, Faculty Of Electronics and Information Technology, Warsaw University of Technology, Warsaw, 2012.

[14] M.H. Habaebi, J. Chebil, A.G. Al-Sakkaf, T. H. Dahawi, comparison between scheduling techniques in long term evolution, IIUM Engineering Journal Vol. 14 No. 1 (2013).

[15] E. Dahlman, S. Parkvall, J. Skold, P. Beming, 3G Evolution: HSPA And LTE for mobile broadband, $2^{\text {nd }}$ Edition, Academic Press, 2008.

[16] R. Jain, The art of computer systems performance analysis: techniques for experimental design, measurement, simulation and modeling, John Wiley \&Sons, New York, 1991.

[17]“LTE System Level Simulator”, Institute Of Communication And Radio Frequency Engineering, Vienna University Of Technology,Vienna.

[18] M. Nejadkheirallah, M. M. Tajari, R. Sookhtsaraei, A. Yousefzadeh, Multi-hop Fuzzy Routing for Wireless Sensor Network with Mobile Sink, Journal of mathematics and computer science Vol 9 (2014) 12-24. 